\title{
Incidencia de la composición de juntas directivas y su grado de formación en el desempeño financiero de las empresas colombianas
}

\author{
Carlos Eduardo Castaño Ríos \\ Universidad de Antioquia \\ Colombia
}

Diana Sirley Tabares Higuita Universidad de Antioquia Colombia

Jair Albeiro Osorio Agudelo Universidad de Antioquia

Colombia

Julián Esteban Zamarra Londoño Universidad de Antioquia Colombia 


\title{
Incidencia de la composición de juntas directivas y su grado de formación en el desempeño financiero de las empresas colombianas
}

\author{
Carlos Eduardo Castaño Ríos ${ }^{(D)}$, Diana Sirley Tabares Higuita ${ }^{(D)}$, Jair Albeiro Osorio \\ Agudelo \& Julián Esteban Zamarra Londoño \\ Universidad de Antioquia - Colombia
}

Para citaciones: Castaño Ríos, C., Tabares Higuita, D., Osorio Agudelo, J. \& Zamarra Londoño, J. (2021). Incidencia de la composición de juntas directivas y su grado de formación en el desempeño financiero de las empresas colombianas. Panorama Económico, 29(3), 172-184.

Recibido: 6 de mayo de 2020

Aprobado: 25 de octubre de 2020

Autor de correspondencia:

Carlos Eduardo Castaño Ríos

eduardo.castano@udea.edu.co

Editor: Andrés Escobar E. Universidad de Cartagena-Colombia.

Tipología IBN Publindex:

Artículo Resultados de Investigación

Científica y Tecnológica

Copyright: () 2021. Castaño Ríos, C., Tabares Higuita, D., Osorio Agudelo, J. \& Zamarra Londoño, J. Este es un artículo de acceso abierto, distribuido bajo los términos de la licencia https://creativecommons.org/licenses/by-nc-

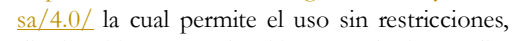
distribución y reproducción en cualquier medio, siempre y cuando que el original, el autor y la fuente sean acreditados.

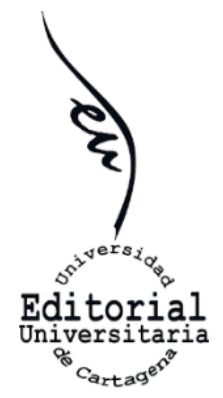

\section{RESUMEN}

El mercado de valores colombiano ha sido poco estudiado desde la perspectiva del Gobierno Corporativo y menos aún, si se considera la composición de las Juntas Directivas de las empresas que cotizan en la Bolsa de Valores. Este artículo busca evidenciar si el nivel de formación y la composición entre externos e internos de las Juntas Directivas inciden en el desempeño financiero de estas entidades. Para tal fin, se emplea una muestra de empresas que cotizan en bolsa y que cuentan con los datos necesarios para el estudio. La información se analizó a través de un modelo de panel de datos y se encuentra que el nivel de formación no tiene relación con el desempeño financiero de las empresas, y que el grado de personas externas (independientes) que están en la organización influyen positivamente en el EBITDA.

Palabras clave: Gobierno Corporativo; EBITDA; Composición Junta Directiva; mercado de valores colombiano.

\section{Impact of the composition of boards of directors and their degree of training on the financial performance of colombian companies}

\begin{abstract}
The Colombian stock market has been little studied from the perspective of Corporate Governance and even less, if the composition of the Boards of Directors of companies listed on the Stock Market is considered. This article seeks to show whether the level of training and the composition with external and internal members of the Boards of Directors affect the financial performance of these entities. For this purpose, a sample of firms that are listed on the stock market was used. The information was analyzed through a data panel model, and it was found that the level of training is not related to the financial performance of the companies, and that the degree of external membership positively influence the EBITDA.
\end{abstract}

Keywords: Corporate Governance; EBITDA; Composition of Board of Directors; Colombian stock market. 


\section{INTRODUCCIÓN}

La creciente necesidad de implementar políticas de Gobierno Corporativo en las empresas, ha llevado a que algunas empresas colombianas generen nuevos reportes que día a día vienen creciendo en información, aunque no sea mucha la aplicabilidad que estas entidades le ven, más allá de estar a la moda con el mercado. De allí, que sea importante indagar sobre si efectivamente algunos de estos reportes como es el caso de la composición de la junta directiva, tienen alguna relación con el desempeño financiero de la entidad.

El mercado de valores colombiano, aunque pequeño, cuenta con importantes volúmenes de transacciones que se vienen convirtiendo en punto de referencia con algunos países latinoamericanos, excepto con México, Brasil y Chile, que ya contaban con una mayor dinámica desde hace años. Sin embargo, este mercado es relativamente nuevo en el contexto internacional, aunque con bastante potencial, puesto que Colombia ha generado crecimientos del PIB importantes y superiores a los de sus similares Latinoamericanos, sin caer aun en períodos de crisis, muy a pesar de lo que sucede a vecinos económicos como Venezuela y Brasil.

Por tal motivo, este trabajo parte de dos grandes objetivos. El primero, demostrar la incidencia que tiene el nivel de formación de los integrantes de las Juntas Directivas sobre el desempeño financiero de las empresas, medido a través del EBITDA. Esta situación, considerando que una formación más elevada de estos integrantes permite a la organización tomar decisiones de mayor calado y mucho más precisas y con ello obtener mejores resultados financieros. En cuanto al segundo, se espera evidenciar que la composición con externos de la Junta Directiva es positiva para el desempeño financiero de la empresa, sin embargo, llega un punto donde este rendimiento empieza a decrecer, lo cual es explicado porque cuando ya no existe quien contradiga esa fuerza mayoritaria, los integrantes externos pueden coludir con la administración y hacer que ya no primen los intereses generales sino los personales.

Para lograr lo anterior, el equipo investigador tomó una muestra de 12 empresas que cotizan en la bolsa de valores de Colombia y que contaban con la respectiva información para hacer el estudio. Pues como se mencionó inicialmente, no todas las empresas colombianas han asumido el rol de hacer este tipo de reportes referentes al Gobierno Corporativo. El análisis con el panel de datos estrictamente balanceado permite evidenciar que el nivel de formación no incide en el resultado financiero de la empresa y que efectivamente, la composición con externos (independientes) sí influye sobre el EBITDA de las empresas, considerando además, que cuando ya el porcentaje de participación de externos supera el 65\% el efecto pasa a ser negativo.

\section{ANTECEDENTES}

\section{Revisión de la literatura}

La literatura plantea algunas teorías que indican que el comportamiento de los directivos sigue sus intereses personales y también, son influenciados por el medio en el que se mueven. Por ejemplo, desde la teoría de la agencia se puede manifestar que 
inversionistas y agentes (gerentes y otros ejecutivos con información privilegiada) tienen intereses contrapuestos, diferente tolerancia al riesgo y acceso a la información de manera asimétrica (Pucheta-Martínez \& Chiva Ortells, 2016). Según, Jensen \& Meckling (2016) quienes gestionan los recursos de la empresa, se aprovecharán de su información privilegiada y seguirán sus intereses propios por encima de lo esperado por los inversionistas, que en la mayor cantidad de los casos, son externos a la administración del negocio. Según esta teoría, la separación entre el control entre la propiedad y el control de la empresa provoca asimetrías de la información entre accionistas y directivos, que generan costos de agencia (Merino et al. 2009). Con la finalidad de contrarrestar estos efectos, los accionistas pueden recurrir a diversos mecanismos de Gobierno Corporativo, tales como las cláusulas contractuales, las estructuras de vigilancia a bordo, e incentivos así mismo, la política de compensación es un mecanismo de control interno que pueden mitigar los costos de agencia y el proceso de pago como medio de alinear los intereses de administración de los accionistas (Shleifer \& Vishny, 1997; Jesen y Meckling 1976; Ozkan 2007a, 2007b).

Por su parte, la teoría de los recursos plantea que las empresas son sistemas abiertos y no solo interactúan con fuerzas institucionales, sino que también realizan transacciones entre sí a nivel de empresa para obtener los recursos necesarios para la supervivencia. Esta teoría hace hincapié en las funciones de provisión de recursos y capacidades de la junta para trabajar hacia la mejora de resultados de la empresa (de Villiers, Naiker, \& van Staden, 2011). Los directores siendo los expertos, tienen experiencia a largo plazo y ocupan posiciones influyentes en otras empresas también. En consecuencia, son una rica fuente de conocimiento y orientación (Pfeffer \& Salancik, 1978) y pueden proporcionar vínculos críticos a los recursos y aprovechar el capital social a través de sus redes sociales (Hillman \& Dalziel, 2003), permitiendo a los administradores adoptar prácticas prosociales específicas que podría ser valioso para mejorar de la empresa (Bansal \& Clelland, 2004; Berrone \& Gómez-Mejía, 2009).

La teoría de los participantes en contraste con la perspectiva de la teoría de la agencia afirma que una empresa tiene relaciones con un conjunto más amplio de partes interesadas, incluidos los empleados, consumidores, gobiernos, defensores del medio ambiente, y otros, más allá de los accionistas (Freeman, 1984). Actúa como una guía para comprender el dominio de las responsabilidades de las empresas (Jamali, Safieddine \& Rabbath, 2008; Parmar et. Al., 2010). A partir de esta idea, la teoría de las partes interesadas (Hill \& Jones, 1992) sugiere que la empresa tiene una relación contractual con todos los interesados, con los administradores en el centro de este nexo, dada su control directo sobre la toma de decisiones. En consecuencia, los sistemas de Gobierno Corporativo deben permitir a las empresas ser gestionados en beneficio de todos sus grupos de interés, tanto financieros como no financieros (de Graaf \& Stoelhorst, 2009), con los directores que tienen un papel fundamental que desempeñan en este sentido.

Según la teoría de la agencia, las juntas son mecanismos para gerentes de supervisión para evitar conflictos de agencia, al mismo tiempo, las juntas representan varios intereses de los participantes en el proceso de toma de decisiones empresariales y cuando son de gran tamaño a menudo se enfrentan a problemas free rider (Dalton, Daily, Ellstrand, \& Johnson, 1998), es decir consumen más que una parte equitativa de un recurso, o se benefician sin entregar una contraprestación. 
La Junta Directiva de una compañía está orientada a tomar decisiones de creación de valor, estrategia de mercado, competitividad, entre otras, sin embargo, los miembros de la Junta tienen diferentes características demográficas, valores y preferencias (Chin, Hambrick, \& Treviño, 2013), que los llevan a actuar de forma distintas. Teorías de la agencia y la administración hacen diferentes hipótesis sobre las motivaciones de gestión, en particular en relación con la selección de los accionistas frente a los intereses de las partes interesadas (Godos-Díez, Fernández-Gago, \& Martínez-Campillo, 2011). Aunque la teoría de la agencia asume la astucia desde la gestión de la organización, la teoría de la administración propone que los directivos pueden ser individuos honestos que puedan adoptar las actividades pro-organizativos y pro-partes interesadas.

También, la forma de actuar puede estar ligada al género del individuo, de acuerdo con la literatura sobre el razonamiento moral (Gilligan, 1982) las mujeres son más sensibles acerca de los escenarios que requieren juicios éticos (Post, Rahman, \& Rubow, 1978). Sin embargo, otras teorías sostienen que las mujeres líderes tienden a ser más innovadores e igualitarias en su visión de la estrategia de la empresa (Glass, Cook, \& Ingersoll, 2015). Resultados neutrales podrían explicarse mediante la invocación de la teoría de la masa crítica (Kramer, Konrad, Erkut, \& Hooper, 2006), que sostiene que los directores mujeres son típicamente directores minoritarios y tienden a convertirse en meras fichas para su grupo (Brewer \& Kramer, 1985). Los cambios de política en algunos países que fomentan una cuota mínima de mujeres en los consejos (por ejemplo, el 40 por ciento en Noruega, Francia, España y los Países Bajos) son propensos a aliviar este problema de la participación simbólica.

Por otra parte, la edad puede ser un favor determinante, los directivos más nuevos y más jóvenes son juzgados por el mercado en relación con su capacidad de entregar resultados financieros (Fabrizi, Mallin, \& Michelon, 2014).

Ahora, para participar y trabajar en pro de la compañía, los ejecutivos reciben una compensación, el cual es un paquete de compensación fija en forma de sueldo, los incentivos financieros a corto plazo en forma de bonificaciones e incentivos a largo plazo, tales como el pago basado en el crecimiento patrimonial (Frye, Nelling, \& Webb, 2006). Las proporciones de estos componentes en el paquete de compensación total de un directivo son determinantes en los conflictos de agencia (Mackenzie, 2007; Zajac \& Westphal, 1994).

Tradicionalmente, una alta proporción de salario base conduce a un arraigo en la administración (Hambrick \& Finkelstein, 1995). Un punto de vista sugiere que para mantener sus posiciones, los gerentes ya establecidos en la empresa pueden adoptar una estrategia de aversión al riesgo (Zajac \& Westphal, 1994). Al mismo tiempo, las estructuras salariales fijas se basan en los objetivos financieros a corto plazo retrospectivos (Mahoney \& Thorn, 2006). Del mismo modo, la teoría de la agencia predice también que una mayor proporción de los pagos de bonificación puede conducir a los ejecutivos a centrarse en consideraciones a corto plazo la línea de fondo (Stata \& Maidique, 1980).

Por otro lado, la literatura académica muestra que la función de la supervisión por parte de las juntas directivas, hacia los CEO se juega por los directores institucionales y no por directores independientes (Pucheta-Martínez y Chiva-Ortells, 2016). Estudios anteriores 
mencionan que los consejeros independientes no realizan el gobierno corporativo y aumentar o no afectan la retribución del CEO (Ozkan 2007a, 2007b), es por eso que es importante analizar el papel de los administradores institucionales en las juntas directivas. Por ejemplo, la presencia de independientes en las juntas es recomendada por el Código de Buen Gobierno de las Sociedades Cotizadas en España (CUBG, 2015) para mejorar la gobernabilidad.

En cuanto a la relación existente entre el nivel de estudios de los integrantes de la junta directiva y su efecto en el desempeño financiero de la empresa, no se encontró literatura específica, lo cual permite identificar un potencial aporte académico sobre el asunto. No obstante, se evidenció un trabajo de Ofe (2012) fue analizada la relación entre el nivel de estudios del CEO y el desempeño financiero de la empresa con una muestra de empresas que cotizan en la bolsa de valores de Estocolmo, para lo cual, los resultados arrojaron que no existía ninguna relación, ni con el tipo de título de pregrado ni con los niveles de posgrados obtenidos por los gerentes con el resultado financiero generado en los distintos períodos.

Por último, vale la pena destacar que el EBITDA es una variable poco usada en la literatura de gobierno corporativo para estudiar el desempeño financiero de las empresas, pues los trabajos, en general concentran su atención sobre variables de resultado final, como la rentabilidad sobre los activos (ROA), la rentabilidad sobre el patrimonio (ROE) o la utilidad neta. Sin embargo, el EBITDA es una medida importante del resultado operativo de la empresa, sin considerar ajustes contables como la amortización y depreciación de activos, lo que genera una base de caja para la empresa, que es en esencia, sobre lo que los gestores empresariales deberían trabajar. Cabe recordar que desde la teoría financiera, las empresas valen por la capacidad que tienen de generar retornos futuros, especialmente, si estos pueden ser medidos en términos de flujos de caja.

\section{Hipótesis}

Las funciones de la Junta Directiva a través de los años y los escándalos presentados, han generado cambios radicales. En algunos estudios se ha analizado la relación entre las características de la Junta Directiva y la compensación de su director general, centrándose principalmente en la composición de la junta y específicamente con la presencia de los directores independientes. Sin embargo, la literatura deja un vacío en relación a si la formación de quienes componen las juntas directivas, afecta o no el desempeño financiero de la empresa.

Entre tanto, esta investigación se centra en determinar si el nivel de la formación de los integrantes de la Junta Directiva, mejora el desempeño del EBITDA, bajo el siguiente postulado:

H1: A mayor nivel de formación de los integrantes de la Junta Directiva, mejor desempeño del EBITDA.

A partir de la idea del trabajo de Pucheta-Martínez y Chiva-Ortells (2016), se puede expresar que muchos de los comportamientos de las Juntas Directivas no son lineales, sino que suelen tener comportamientos en $U$ o en $U$ invertida, dependiendo de las 
variables que son analizadas, con lo cual, se tienen modelos cuadráticos para el análisis. Por ejemplo, cuando los integrantes de algunas características (género, institucional, externos) de las Juntas ya superan en cierta medida un porcentaje de participación dentro de la misma, sus comportamiento tienden a ser colusivos y por ende, se pierde la efectividad de su control para la toma de decisiones.

Bajo la premisa planteada, es de vital importancia considerar que quienes componen la Junta Directiva requieren ser de diversas naturalezas y se puede expresar que cuando la Junta se compone por externos, las decisiones llegan a ser más objetivas, puesto que no laboran al interior de la organización y pueden separar fácilmente los intereses personales y sobreponer los intereses de los terceros que representan con la finalidad de obtener el máximo rendimiento del patrimonio de la organización. Sin embargo, es de considerar que en efecto, puede llegar un punto en el cual los externos superan en gran medida a los internos y ya no busquen optimizar el resultado de los rendimientos de la organización, sino que caigan en la colusión en contravía de los intereses de la empresa para obtener más rendimiento personal.

En tal sentido se plantea la siguiente hipótesis:

H2: A mayor participación de externos en la Junta Directiva mejor desempeño del EBITDA, pero llega un punto donde el comportamiento es inverso.

\section{METODOLOGÍA Muestra}

Para realizar el estudio se consideran las empresas colombianas que cotizan en la Bolsa de Valores de Colombia para el periodo de 2010 al 2014 que se encuentran en el índice Accionario de capitalización COLCAP, indicador que refleja las variaciones de los precios de las 20 acciones más liquidas de la Bolsa de Valores de Colombia (BVC), donde el valor de la capitalización bursátil ajustada de cada compañía determina su participación dentro el índice.

Inicialmente al revisar el indicador se encuentran efectivamente las 20 acciones más liquidas, sin embargo al realizar un análisis de las acciones se evidencia que algunas de las empresas participan con acciones preferenciales y ordinarias, lo cual hace que se disminuya la muestra, así mismo algunas de las empresas no disponen de la información completa requerida para el estudio, quedando finalmente para el trabajo de análisis con 12 empresas, que se muestran en la tabla 1.

Tabla 1. Empresas de la muestra

\begin{tabular}{|c|l|}
\hline \multicolumn{2}{|c|}{ EMPRESAS DEL COLCAP } \\
\hline EMPRESA & \multicolumn{1}{c|}{ BREVE DESCRIPCIÓN } \\
\hline Grupo Argos & $\begin{array}{l}\text { Compañía holding con inversiones sostenibles en sectores estratégicos de la } \\
\text { infraestructura: cemento, energía, puertos, carbón e inmobiliario. }\end{array}$ \\
\hline Avianca Holdings S.A, & $\begin{array}{l}\text { Compañía Holding, bajo la cual se integran, entre otras compañías, varias } \\
\text { empresas aéreas con operación doméstica e internacional. }\end{array}$ \\
\hline Grupo Bancolombia & $\begin{array}{l}\text { Es una organización financiera colombiana, perteneciente al Grupo Sura, a } \\
\text { su vez parte del Grupo Empresarial Antioqueño, es el banco privado más } \\
\text { grande del país (por el tamaño de su patrimonio y activos) y uno de los más } \\
\text { grandes de América }\end{array}$ \\
\hline
\end{tabular}




\begin{tabular}{|c|c|}
\hline \multicolumn{2}{|r|}{ EMPRESAS DEL COLCAP } \\
\hline EMPRESA & BREVE DESCRIPCIÓN \\
\hline $\begin{array}{l}\text { Bolsa de Valores de } \\
\text { Colombia BVC }\end{array}$ & $\begin{array}{l}\text { Es el principal foro de negociación y registro de operaciones sobre valores } \\
\text { en Colombia, es una compañía privada listada en el mercado de valores. La } \\
\text { BVC es una bolsa multi-producto y multi-mercado }\end{array}$ \\
\hline Celsia S.A.E.S.P. & $\begin{array}{l}\text { Empresa de energía que ha redefinido su estrategia alrededor del cliente, } \\
\text { ampliando su portafolio a tres nuevos negocios: Ciudades-Sostenibilidad, } \\
\text { Empresas-Productividad y Hogares-Bienestar, en línea con las nuevas } \\
\text { tendencias y necesidades del mercado eléctrico. }\end{array}$ \\
\hline Cementos Argos & Es una empresa de industria cementera colombiana. \\
\hline Ecopetrol S.A. & $\begin{array}{l}\text { Ecopetrol es una empresa de economía mixta, de carácter comercial, } \\
\text { organizada bajo la forma de sociedad anónima, del orden nacional, } \\
\text { vinculada al Ministerio de Minas y Energía, de conformidad con lo } \\
\text { establecido en la Ley } 1118 \text { de 2006, regida por los Estatutos Sociales que se } \\
\text { encuentran contenidos de manera integral en la Escritura Pública n. } 5314 \\
\text { del } 14 \text { de diciembre de 2007, otorgada en la Notaría Segunda del Círculo } \\
\text { Notarial de Bogotá. Es la primera compañía de petróleo de Colombia. }\end{array}$ \\
\hline Grupo Éxito & $\begin{array}{l}\text { El Grupo Éxito se ha transformado en una corporación multiindustria, } \\
\text { multiformato, multimarca y multinegocio, que está compuesta por su } \\
\text { empresa matriz Almacenes Éxito S.A. y sus filiales Distribuidora de Textiles y } \\
\text { Confecciones S.A., Didetexco S.A., Almacenes Éxito Inversiones S.A.S. y } \\
\text { Carulla Vivero Holding Inc; y dentro de su propuesta opera, además del } \\
\text { comercio al retail, otras ocho industrias: inmobiliaria, financiera, seguros, } \\
\text { textiles, alimentos, viajes, telefonía móvil y estaciones de servicio }\end{array}$ \\
\hline $\begin{array}{c}\text { Interconexión Eléctrica } \\
\text { S.A. E.S.P. - ISA }\end{array}$ & $\begin{array}{l}\text { El holding estatal colombiano, a través de sus filiales, participa en los sectores } \\
\text { de energía, telecomunicaciones y concesiones viales en Latinoamérica; } \\
\text { focaliza sus actividades en los negocios de Transporte de Energía Eléctrica, } \\
\text { Transporte de Telecomunicaciones, Concesiones Viales y Gestión Inteligente } \\
\text { de Sistemas de Tiempo Real. }\end{array}$ \\
\hline ISAGEN & Empresa de generación y la comercialización de soluciones energéticas. \\
\hline Grupo Nutresa & $\begin{array}{l}\text { Grupo Nutresa S.A., nombre que recoge todas las categorías de alimentos } \\
\text { del grupo y fortalece el vínculo de todas sus marcas con la salud, la nutrición } \\
\text { y el bienestar. }\end{array}$ \\
\hline $\begin{array}{c}\text { Grupo de inversiones } \\
\text { Suramericana }\end{array}$ & $\begin{array}{l}\text { Somos una compañía de origen colombiano y con proyección internacional, } \\
\text { cuyo propósito es promover sinergias e identificar nuevas oportunidades de } \\
\text { creación, crecimiento y expansión de negocios, con base en la innovación } \\
\text { entre las compañías que hacen parte de nuestro portafolio, las cuales se } \\
\text { encuentran principalmente en los sectores de servicios financieros, seguros, } \\
\text { seguridad social y servicios complementarios. Así mismo, contamos con } \\
\text { importantes inversiones en los sectores de cementos yalimentos, entre otros. }\end{array}$ \\
\hline
\end{tabular}

Fuente: Elaboración propia, a partir de informes de gestión de las empresas.

Es importante aclarar que la información financiera fue extraída de los informes integrados de cada una de las empresas año por año para el periodo de análisis. Adicionalmente, como se evidencia en la tabla 1, las empresas son de diferentes sectores económicos, para el estudio no se excluye el sector financiero ya que se considera que el sector económico no influye en las variables a analizar.

\section{Modelo empírico}

La estimación se realiza mediante un modelo log-lineal para disminuir la dispersión original de la variable dependiente (EBITDA en billones de pesos); el modelo de panel de datos estimado es el siguiente: 


\section{$\log \left(y_{i}\right)=\beta_{i}+\beta_{2} x_{2}+\beta_{3} x_{3}+u_{i}$}

Donde $\log \left(y_{i}\right)$ es el logaritmo natural del EBITDA en billones de la empresa i, $x_{2}$ es el porcentaje de miembros independientes en la junta directiva de la empresa i, $x_{3}$ es el porcentaje que cuenta con posgrado y finalmente $u_{i}$ es el término de error.

En este sentido, la variable dependiente a evaluar es el EBITDA, medida en billones de pesos para cada una de las empresas. Se determina este indicador financiero para determinar el nivel de beneficios que tienen las empresas en su actividad económica. Por otro lado, se determina que las variables independientes son el nivel de formación y nivel de independencia de los miembros de la Junta Directiva de las empresas seleccionadas. Se considera nivel de formación pos gradual y el nivel de independencia como miembros internos y externos.

\section{RESULTADOS}

La base de datos es un panel estrictamente balanceado (mismo número de variables para igual número de años en todos los grupos), la conforman 12 empresas con la respectiva composición de sus juntas directivas por nivel de educación y los resultados del EBITDA en el periodo 2010-2014. En la tabla 2 se pueden evidenciar los datos descriptivos arrojados en el primer análisis de la muestra.

Tabla 2. Estadísticos Descriptivos

Estadísticas descriptivas

\begin{tabular}{llllllll}
\hline Variable & \multicolumn{1}{c}{ Media } & \multicolumn{2}{l}{ Desviación estándar } & Min & Max & Observacione \\
Ebitda en billones & & & & & & \\
& General & 2,95 & 6.629 .704 & .0000309 & 28.5 & $\mathrm{~N}=$ & 60 \\
& Entre empresas & & 6.649 .858 & .0000358 & 23.96 & $\mathrm{n}=$ & 12 \\
& En la empresa & & 1.652 .382 & -640.983 & 749.017 & $\mathrm{~T}=$ & 5 \\
& & & & & & \\
Porcentaje independientes & General & .5493333 & .1240266 & .29 & .71 & $\mathrm{~N}=$ & 60 \\
& Entre empresas & & .1081256 & .33 & .71 & $\mathrm{n}=$ & 12 \\
& En la empresa & & .0669632 & .2133333 & .6693333 & $\mathrm{~T}=$ & 5 \\
& & & & & & \\
Porcentaje posgrados & General & .8245 & .1788895 & .36 & 1 & $\mathrm{~N}=$ & 60 \\
& Entre empresas & & .173073 & .386 & 1 & $\mathrm{n}=$ & 12 \\
& En la empresa & .063859 & .5925 & 10.005 & $\mathrm{~T}=$ & 5 \\
\hline
\end{tabular}

Fuente: Elaboración propia

La estimación se realizó mediante un modelo log-lineal para disminuir la dispersión original de la variable dependiente (EBITDA en billones de pesos colombianos); el modelo de panel de datos estimado fue el siguiente:

$$
\log \left(y_{i}\right)=\beta_{i}+\beta_{2} x_{2}+\beta_{3} x_{3}+u_{i}
$$

Donde $\log \left(y_{i}\right)$ es el logaritmo natural en billones de la empresa i, $x_{2}$ es el porcentaje de miembros independientes en la junta directiva de la empresa $i, x_{3}$ es el porcentaje que cuenta con posgrado y finalmente $u_{i}$ es el término de error.

Según los resultados observados en la tabla 3, los modelos log-lineales estimados 1 y 4 muestran significancia estadística positiva del porcentaje de miembros independientes en la junta directiva de la empresa sobre la dinámica del EBITDA. Para ambos modelos, manteniendo todo lo demás constante, aumentos de $1 \%$ en el porcentaje de integrantes 
independientes se traduce en aumentos de aproximadamente 4.3\% en el EBITDA de la empresa. No obstante, si se observa el gráfico relación ebitda-\% de independientes en la junta directiva (ver gráfico 1), la relación llega a un límite alrededor del 65\% en el cual el efecto empieza a desaparecer.

Tabla 3. Resultados del modelo

\begin{tabular}{|c|c|c|c|c|c|c|}
\hline VARIABLES & $\begin{array}{c}\text { (1) EA } \\
\text { Modelo_1 } \\
\text { In_ebitda }\end{array}$ & $\begin{array}{c}\text { (2) } \\
\text { Modelo_2 } \\
\text { In_ebitda } \\
\end{array}$ & $\begin{array}{c}\text { (3) } \\
\text { Modelo_3 } \\
\text { In_ebitda }\end{array}$ & $\begin{array}{c}\text { (4) EF } \\
\text { Modelo_4 } \\
\text { In_ebitda }\end{array}$ & $\begin{array}{c}\text { (5) } \\
\text { Modelo_5 } \\
\text { In_ebitda } \\
\end{array}$ & $\begin{array}{c}\text { (6) } \\
\text { Modelo_6 } \\
\text { In_ebitda }\end{array}$ \\
\hline Porcentaje independientes & $\begin{array}{l}4.340 * \\
(2.400)\end{array}$ & & $\begin{array}{c}4.342 \\
(3.682)\end{array}$ & $\begin{array}{l}4.294^{*} \\
(2.535)\end{array}$ & $\begin{array}{c}1.238 \\
(28.719)\end{array}$ & $\begin{array}{l}79.242^{\star \star} \\
(38.441)\end{array}$ \\
\hline Porcentaje Posgrado & & $\begin{array}{l}0.250 \\
(2.441)\end{array}$ & $\begin{array}{l}-0.095 \\
(1.601)\end{array}$ & $\begin{array}{l}-0.944 \\
(2.658)\end{array}$ & & \\
\hline Porcentaje independientes ^2 & & & & & $\begin{array}{c}3.013 \\
(27.746)\end{array}$ & $\begin{array}{l}-59.752 * \\
(34.482)\end{array}$ \\
\hline Constant & $\begin{array}{l}-3.136^{*} \\
(1.628)\end{array}$ & $\begin{array}{l}-0.958 \\
(2.225)\end{array}$ & $\begin{array}{l}-3.059 \\
(2.195)\end{array}$ & $\begin{array}{l}-2.333 \\
(2.535)\end{array}$ & $\begin{array}{l}-2.387 \\
(7.117)\end{array}$ & $\begin{array}{c}-26.184^{\star \star} \\
(10.597)\end{array}$ \\
\hline Observations & 60 & 60 & 60 & 60 & 60 & 51 \\
\hline Number of groups & 12 & 12 & 12 & 12 & 12 & 11 \\
\hline
\end{tabular}

Fuente: Elaboración propia

Para la estimación del modelo 1 se emplearon efectos aleatorios para controlar la variabilidad entre los grupos de empresas, mientras que en el modelo 4 se controló la variabilidad al interior del grupo con el uso de efectos fijos. Como se mencionó anteriormente, en ambos casos fue positivo y significativo el porcentaje de miembros independientes sobre el EBITDA. En modelo 3, cuando se usaron las variables de forma conjunta con efectos aleatorios, el hallazgo no fue estadísticamente significativo.

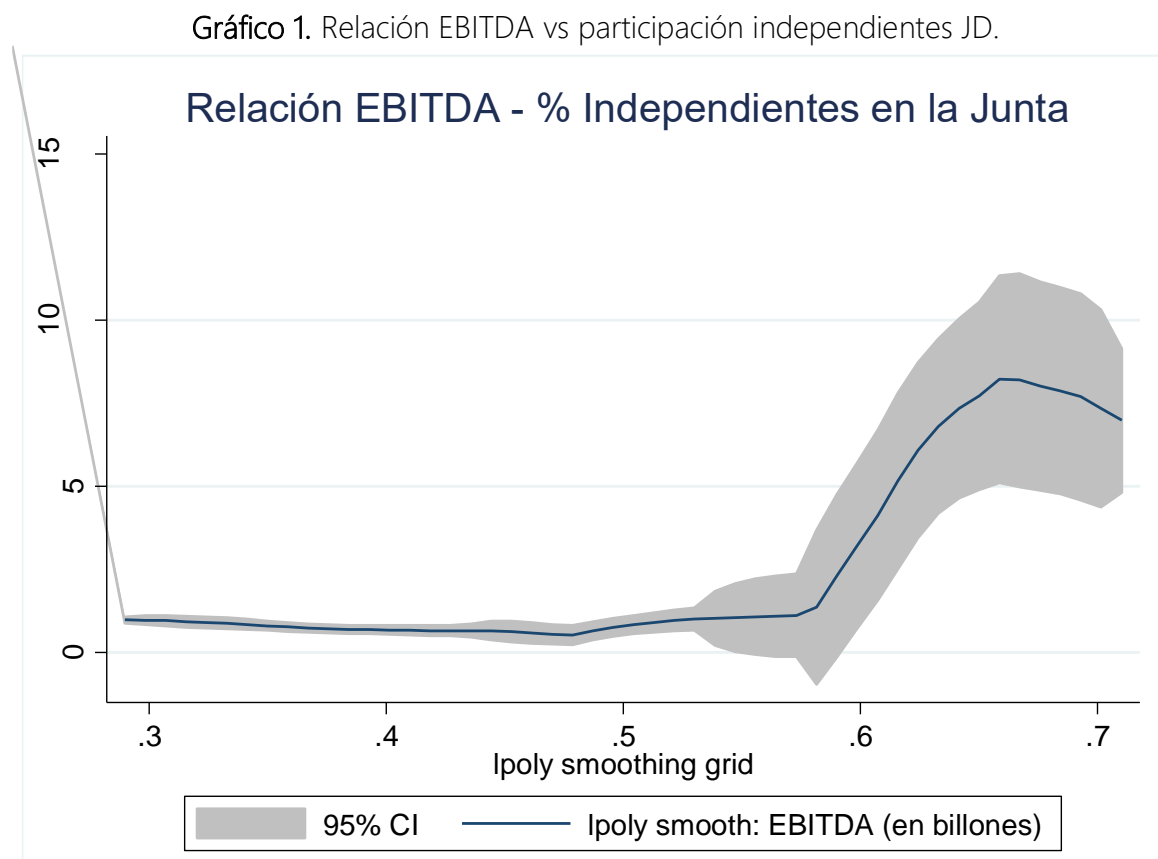


En cuanto al modelo 4, en el cual también se utiliza como variable de control el porcentaje de integrantes de la junta directiva que cuenta posgrado, se observa un efecto negativo aunque no significativo sobre la variable dependiente.

En la estimación número 2, se utiliza como variable independiente el porcentaje de integrantes de la junta directiva con posgrado; aunque el coeficiente es positivo, la relación observada es no significativa.

Otros ejercicios incluyeron el uso como regresores del porcentaje de miembros de la junta directiva con maestría y doctorado, aunque se rechazaba la hipótesis nula de que tuviera impacto sobre el desempeño de la empresa. Para el caso del modelo del modelo 5 se utilizó el total de la muestra, sin obtener resultados estadísticamente significativos, aunque con un dato que se consideró podría afectar el resultado por su baja participación de independientes. Mientras, para el modelo 6 se realizó para empresas con porcentajes mayores a 40\%, de esta manera se saca de la muestra la empresa con menor participación de independientes en la junta directiva (ver gráfico 1), con lo cual, se obtiene el signo positivo del primer regresor que indica el efecto positivo y estadísticamente significativo sobre el desempeño del EBITDA. En el segundo regresor, el signo negativo indica la forma concava de esta variable, esto es, a un mayor número de integrantes independientes el efecto sobre el EBITDA es cada vez menor.

\section{CONCLUSIONES}

En Colombia, no se encuentra evidencia presente sobre trabajos académicos de Gobierno Corporativo que permitan establecer criterios para el mejoramiento al futuro de las políticas de Gobierno Corporativo que tienen las empresas que cotizan en bolsa. Este primer acercamiento permite dejar al menos unos primeros resultados en torno a la importante incidencia que tiene la participación de personas externas (independientes) en las Juntas Directivas de estas grandes organizaciones colombianas. No obstante, se reitera lo que ha sucedido en otros países como España (Pucheta-Martínez \& ChivaOrtells, 2016), donde llega un punto en el cual la participación excesiva de algún tipo de integrante, consigue afectar el proceso de toma de decisiones, en este caso, se encontró que dicho punto está cercano al $65 \%$ de externos, es decir, las Juntas Directivas no podrán componerse en más de este porcentaje, con miras a maximizar su EBITDA.

Asimismo, se deja evidencia para el caso colombiano, sobre el nivel de formación de los integrantes de las Juntas Directivas y su efecto en el desempeño financiero, en este trabajo, del EBITDA, para lo cual se obtiene que no existe una relación entre tales variables. Frente a esta situación, será valioso que en futuros trabajos, se pueda analizar si lo que realmente pueda incidir tiene que ver con los años de experiencia en general de los miembros de junta, o si es su cercanía con industrias de la cadena de valor, o su experiencia específica en el sector, inclusive, analizar si ha tenido alguna relación con el sector público y si esto en efecto, incide en el desempeño observado de la variable financiera.

En relación a las limitaciones, se debe señalar que la muestra es bastante pequeña, sin embargo, es significativa para el mercado de valores colombiano, donde estas mismas entidades, son las que regularmente mantienen movimiento en la bolsa de valores y son 
las que afectan el desempeño de los índices del mercado colombiano. Asimismo, este trabajo solo es aplicable para el caso colombiano, aunque puede ser de utilidad como base para futuros trabajos en el país sobre gobierno corporativo y en especial, de las juntas directivas y su incidencia en los resultados financieros de las empresas que están en la bolsa de valores. Por último, es bastante limitada la información que sobre Gobierno Corporativo reportan las empresas colombianas, por lo cual, será importante seguir insistiendo sobre la importancia de este tipo de reportes en el país con miras a una participación clave en el mercado global y para el mejoramiento de los indicadores actuales a través de la investigación.

Como aporte se considera que es un primer acercamiento al mercado colombiano desde la mirada del Gobierno Corporativo y las juntas directivas empresariales. Además, la variable de desempeño financiero EBITDA puede permitir profundizar el estudio sobre el potencial crecimiento de las empresas, gracias a que este es un indicador financiero clave al momento de generar valor y flujos de efectivo en las empresas.

\section{REFERENCIAS}

Bansal, P., \& Clelland, I. (2004). Talking trash: Legitimacy, impression management, and unsystematic risk in the context of the natural environment. Academy of Management Journal, 47:93-103.

Bolsa de valores de Colombia (2011). METODOLOGÍA PARA EL CÁLCULO DEL ÍNDICE COLCAP. Extraído el 20 de julio de 2016 de http://www.bvc.com.co/recursos/Files/Mercados/Indices Accionarios/Nueva Metodologia Calc ulo Indice COLCAP.pdf

Berrone, P., \& Gómez Mejía, L. (2009). Environment performance and executive compensation: An integrated agency-institutional perspective. Academy of Management Journal, 52: 103-26.

Brewer, M. B., \& Kramer, R. M. (1985). The psychology of intergroup attitudes and behaviour. Annual Review of Psychology, 36:219-43.

Chin, M., Hambrick, D., \& Treviño, L. (2013). Political ideologies of CEOs: The influence of executives' values on corporate social responsability. Administrative Science Quarterly, 58:197-232.

CUBG 2015. Código de Buen Gobierno de las Sociedades Cotizadas (Conthe Code). Comisión Nacional del Mercado de Valores (CNMV), www.cnmv.es

Dalton, D. R., Daily, C. M., Ellstrand, A. E., \& Johnson, J. L. (1998). Meta-analytic reviews of board composition, leadership structure, and financial performance. Strategic Management Journal, 19:269-90.

de Graaf, F. J., \& Stoelhorst, W. (2009). The role of governance in corporate social responsibility: Lessons from Dutch finance. Bussines \& Society, 52:282-317.

de Villiers, C., Naiker, V., \& van Staden, C. J. (2011). The effect of board characteristics on firm enviromental performance. Journal of Management, 37: 1636-63.

Fabrizi, M., Mallin, C., \& Michelon, G. (2014). The role of CEO's personal incentives in driving corporate social responsibility. Journal of Business Ethics, 124:311-26.

Freeman, R. (1984). Strategicmanagement: A stakeholder approach. Boston: MA:Pitman.

Frye, M. B., Nelling, E., \& Webb, E. (2006). Executive compensation in socially responsable firms. Corporate Governance: An International Review, 14: 446-55. 
Gilligan, C. (1982). In a different voice: Psychological theory and women's development. Cambridge: MA: Harvard University Press.

Glass, C., Cook, A., \& Ingersoll, A. R. (2015). Do women leaders promote sustainability? Analyzing the effect of corporate governance composition on environmental performance. Business Strategy and the Environment, DOI:10.1002/bse. 1879.

Godos-Díez, J. L., Fernández-Gago, R., \& Martínez-Campillo, A. (2011). How important are CEOs to CSR practices? An analysis of the mediating effect of the perceived role of ethics and social responsibility. Journal of Business Ethics, 98:531-48.

Granovetter, M. (1985). Economic action and social structure: The problem of embeddedness. American Journal of Sociology, 91: 481-510.

Hambrick, D. C., \& Finkelstein, S. (1995). The effects of ownership structure on conditions at the top: The case ofCEO pay raises. Strategy Management Journal, 16: 175-94.

Hill, C. W., \& Jones, T. M. (1992). Stakeholder agency theory. Academy of Management Studies, 29:131-54.

Hillman, A. J., \& Dalziel, T. (2003). Boards of directors and firm performance: Integrating agency and resource dependence perspectives. Academy of Management REview, 28: 383-96.

Jamali, D., Safieddine, A., \& Rabbath, M. (2008). Corporate governance and corporate social responsibility: Synergies and interrelationships. Corporate Governance: An International Review, 16:443-59.

Jensen, M. C., \& Meckling, W. (1976). Theory of the firm:Managerial behaviour, agency costs and ownership structure. Journal of Financial Economics, 3:305-60.

Kramer, V., Konrad, A., Erkut, S., \& Hooper, M. J. (2006). Critical mass on corporate boards:Why three ormore women enhance governance. Wellesley: MA: Wellesley Centers for Women.

Mackenzie, C. (2007). Boards, incentives and corporate social responsibility: The case for a change of emphasis. Corporate Governance: An international review, 15: 935-43.

Mahoney, L. S., \& Thorn, L. (2006). An examination of the structure of executive compensation and corporate social responsibility: A Canadian investigation. Journal of Business Ethics, 64: 149-62.

Merino, E., Manzaneque, M. \& Banegas, R. (2009). Retribución y composición del consejo de administración. Evidencia empírica para las empresas cotizadas españolas. Pecvnia, 8: 203-234.

Ofe, H. A. (2012). Chief Executive Officer's (CEO's) Educational Background and Firm Performance: An empirical study on Manufacturing and IT listed firms in the Stockholm Stock Exchange. Umeå University, Faculty of Social Sciences, Umeå School of Business and Economics (USBE). Independent thesis Advanced level (degree of Master (Two Years))

Ozkan, N. (2007a). CEO compensation and firm performance: an empirical investigation of UK panel data. http://papers.ssrn.com/sol3/papers.cfm?abstract id=1102703 Posted date: March 2008.

Ozkan, N. (2007b). Do corporate governance mechanims influence CEO compensation? An empirical investigation of UK companies. Journal of Multinational Financial Management, 17(5): 349-364.

Parmar, B. L., Freeman, R. E., Harrison, J. S., Wicks, A. C., Purnell, L., \& de Colle, S. (2010). Stakeholder theory: The state of the art. Academy of Management Annuals, 4:403-45.

Pfeffer, J., \& Salancik, G. R. (1978). The external control of organizations: A resource dependence perspective. New York: Harper \& Row. 
Post, C., Rahman, N., \& Rubow, E. (1978). The external control of organizations: A resource dependence perspective. New York: Harper \& Row.

Pucheta-Martínez, M. C. \& Bel-Oms, I. (2016). Institutional female directors on boards and firm value. Journal of Business Ethics, in press.

Pucheta-Martínez, M. C. \& Chiva-Ortells, C. (2016). CEO compensation and board of directors: the role of different types of institutional directors. Inédito.

Shleifer, A., \& Vishny, R. (1997). A survey of corporate governance. The Journal of Finance, 52:73783.

Stata, R., \& Maidique, M. (1980). Bonus system for balanced strategy. Harvard Business Review, 58 : $156-63$.

Zajac, E. J., \& Westphal, J. D. (1994). The costs and benefits of managerial incentives and monitoring in large U.S. corporations: When is more not better? Strategic Management Journal, 15:121-42. 